Copyright by the American Institute of Physics (AIP). Harikrishna, Hari; Ducker, William A.; Huxtable, Scott T., "The

influence of interface bonding on thermal transport through solid-liquid interfaces," Appl. Phys. Lett. 102, 251606 (2013); http://dx.doi.org/10.1063/1.4812749

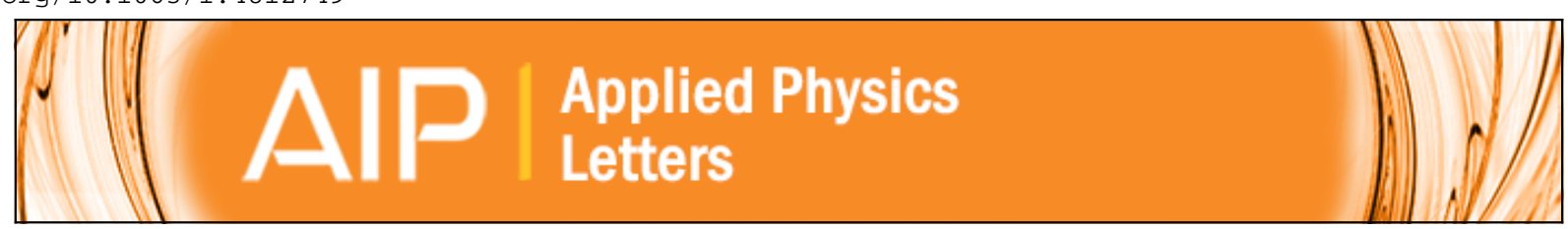

The influence of interface bonding on thermal transport through solid-liquid interfaces

Hari Harikrishna, William A. Ducker, and Scott T. Huxtable

Citation: Applied Physics Letters 102, 251606 (2013); doi: 10.1063/1.4812749

View online: http://dx.doi.org/10.1063/1.4812749

View Table of Contents: http://scitation.aip.org/content/aip/journal/apl/102/25?ver=pdfcov

Published by the AIP Publishing

AlPP Re-register for Table of Content Alerts 


\title{
The influence of interface bonding on thermal transport through solid-liquid interfaces
}

\author{
Hari Harikrishna, ${ }^{1}$ William A. Ducker, ${ }^{2}$ and Scott T. Huxtable ${ }^{3, a)}$ \\ ${ }^{1}$ Department of Engineering Science and Mechanics, Virginia Tech, Blacksburg, Virginia 24061, USA \\ ${ }^{2}$ Department of Chemical Engineering, Virginia Tech, Blacksburg, Virginia 24061, USA \\ ${ }^{3}$ Department of Mechanical Engineering, Virginia Tech, Blacksburg, Virginia 24061, USA
}

(Received 27 April 2013; accepted 16 June 2013; published online 27 June 2013)

\begin{abstract}
We use time-domain thermoreflectance to show that interface thermal conductance, $G$, is proportional to the thermodynamic work of adhesion between gold and water, $W_{\mathrm{SL}}$, for a series of five alkane-thiol monolayers at the gold-water interface. $W_{\mathrm{SL}}$ is a measure of the bond strength across the solid-liquid interface. Differences in bond strength, and thus differences in $W_{\mathrm{SL}}$, are achieved by varying the terminal group ( $\omega$-group) of the alkane-thiol monolayers on the gold. The interface thermal conductance values were in the range $60-190 \mathrm{MW} \mathrm{m}^{-2} \mathrm{~K}^{-1}$, and the solid-liquid contact angles span from $25^{\circ}$ to $118^{\circ}$. (C) 2013 AIP Publishing LLC.
\end{abstract}

[http://dx.doi.org/10.1063/1.4812749]

For sufficiently small materials and devices, thermal transport can be measurably affected by interface conductance, or even the conductance of a single bond. This effect is growing in technological importance as characteristic lengthscales of structures and devices continue to get progressively smaller. ${ }^{1-3}$ For example, interfacial thermal transport at a nanometer scale is important in molecular electronics and conventional integrated circuits, ${ }^{1,4,5}$ polymeric composites used in the air-vehicle industry, ${ }^{6}$ layered structures for thermal barrier coatings, ${ }^{7}$ nanostructured thermoelectric materials, ${ }^{8-10}$ and could play a role in the eventual development of thermal rectifiers. ${ }^{11,12}$ Interface thermal conductance is also important at solid-liquid interfaces for cases including nanoparticle medical therapies, ${ }^{13,14}$ evaporation, ${ }^{15}$ spray cooling, ${ }^{16}$ and electrochemistry. ${ }^{17,18}$ In this letter, we examine the fundamental mechanisms that control heat transfer at solid-liquid interfaces through measurements of the interface thermal conductance as a function of the bonding between an organic film and water.

A heat flux normal to an interface creates a temperature drop, $\Delta T$, across the interface. For a heat flux, $J_{Q}$, across an interface, the interface thermal conductance, $G$, can be quantified as $G=-J_{Q} / \Delta T$. Using this definition, all of the thermal characteristics of the interface are lumped into this single parameter in much the same way as the "slip length" lumps all momentum transport or the "surface excess" lumps all excesses of material. Since many different heat transfer mechanisms may contribute to $G$, it is often difficult to properly interpret experiments to determine which mechanisms dominate. Thus, it is necessary in experiments to control the interfacial properties so that only a limited number of variables operate.

Following in the footsteps of our predecessors, ${ }^{19,20}$ we control the characteristics of the solid-liquid interface through preparation of a series of self-assembled monolayers (SAMs) on the solid. We use gold as the solid because of the facile preparation of well-ordered thiol SAMs on gold, ${ }^{21}$ and we maintain a constant monolayer structure, varying only

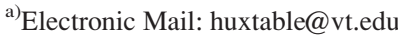

the $\omega$-group (the terminal group of the thiol), with one exception. The structures of the molecules are shown in Table I. Our aim is to vary the strength of the bonding between the solid and liquid and to examine the effect of bonding on $G$, with the hypothesis that stronger bonding produces greater interface conductance. The simple-minded mechanism is that stronger bonding of the solid to the liquid enables more efficient transfer of vibrational energy from the solid to the liquid.

Our work follows the pioneering study by Ge et al., ${ }^{19}$ where they found that a single monolayer of material at a solid-water interface caused a dramatic change in interface conductance. They compared $G$ for hydrophobic SAMs prepared from octadecyltrichlorosilane (OTS) to hydrophilic SAMs prepared from 2-\{methoxy(polyethyleneoxy)-proply\}trichlorosilane (PEG-silane) at the interface between water and the native oxide on aluminum. They measured $G \sim 60 \mathrm{MW}$ $\mathrm{m}^{-2} \mathrm{~K}^{-1}$ on the hydrophobic SAM, and $G \sim 180 \mathrm{MW} \mathrm{m}^{-2}$ $\mathrm{K}^{-1}$ on the hydrophilic SAM. A similar trend was observed for SAMs at the interface between water and gold. Here, $G$ was only $\sim 50 \mathrm{MW} \mathrm{m}{ }^{-2} \mathrm{~K}^{-1}$ for a hydrophobic SAM produced from adsorption of 1-octadecanethiol $\left(\mathrm{C}_{18}\right)$, whereas $G$ was $\sim 100 \mathrm{MW} \mathrm{m}^{-2} \mathrm{~K}^{-1}$ for a hydrophilic SAM produced from adsorption of 11-mercapto-1-undecanol $\left(\mathrm{C}_{11} \mathrm{OH}\right)$. Thus, both comparisons showed greater interface conductance on a hydrophilic film than on a hydrophobic film.

Shenogina et $_{\text {al. }}{ }^{20}$ used molecular dynamics (MD) simulations to examine how the $\omega$-group of alkane-thiols affected $G$ for the gold-water system. They determined both the interface conductance and the water contact angle, $\theta$, from the simulations. Their results showed that $G$ was proportional to $(1+\cos \theta)$ for $\theta$ between $60^{\circ}$ and $115^{\circ}$, and $G$ was roughly constant for $15^{\circ}$ and $60^{\circ}$. They explained the proportionality using the thermodynamic work of adhesion, $W_{\mathrm{SL}}$, which is the minimum work required to separate the liquid from the solid in vapor

$$
\begin{aligned}
& W_{S L}=\gamma_{S V}+\gamma_{L V}-\gamma_{S L}, \\
& W_{S L}=\gamma_{L V}(1+\cos \theta),
\end{aligned}
$$


TABLE I. Molecules and water contact angles for the preparation of self-assembled monolayers.

\begin{tabular}{|c|c|c|c|c|}
\hline Molecular constituent of SAM & SAM molecule & SAM abbreviation & Contact Angle $^{\mathrm{a}}$ & $G^{\mathrm{b}}\left(\mathrm{MW} \mathrm{m}^{-2} \mathrm{~K}^{-1}\right)$ \\
\hline $\mathrm{HSC}_{10} \mathrm{H}_{20}-\mathrm{CH}_{3}$ & $n$-undecanethiol & $\omega-\mathrm{CH}_{3}$ & $\begin{array}{l}\text { Adv: } 118 \pm 2^{\circ} \\
\text { Rec: } 106 \pm 2^{\circ}\end{array}$ & $65 \pm 5$ \\
\hline $\mathrm{HSC}_{2} \mathrm{H}$ & Methyl 3-mercaptopropionate & $\omega$-ester & $\begin{array}{l}\text { Adv: } 76 \pm 2^{\circ} \\
\text { Rec: } 68 \pm 3^{\circ}\end{array}$ & $140 \pm 15$ \\
\hline $\mathrm{HSC}_{11} \mathrm{H}_{22}$ & 11-(1H-pyrrol-1-yl) undecane-1-thiol & $\omega$-pyrrol & $\begin{array}{l}\text { Adv: } 78 \pm 3^{\circ} \\
\text { Rec: } 70 \pm 2^{\circ}\end{array}$ & $140 \pm 15$ \\
\hline $\mathrm{HSC}_{11} \mathrm{H}_{22}$ & 11-Mercapto-1-undecanol & $\omega-\mathrm{OH}$ & $\begin{array}{l}\text { Adv: } 25 \pm 2^{\circ} \\
\text { Rec: } 20 \pm 2^{\circ}\end{array}$ & $190 \pm 30$ \\
\hline $\mathrm{HSC}_{10} \mathrm{H}_{20}$ & 11-Mercapto-undecanoic acid & $\omega-\mathrm{COOH}$ & $\begin{array}{l}\text { Adv: } 27 \pm 3^{\circ} \\
\text { Rec: } 17 \pm 2^{\circ}\end{array}$ & $190 \pm 30$ \\
\hline
\end{tabular}

${ }^{a}$ Angles are the measured advancing (Adv) and receding (Rec) contact angles of water on the monolayer in air. The uncertainty values listed for the contact angles span the range of values observed on repeated measurements on multiple films.

${ }^{\mathrm{b}}$ The uncertainty values for $G$ represent the range of values that could fit our experimental data given the propagation of uncertainties in our experimental data and in the parameters that are input to our thermal model (e.g., metal film thicknesses and conductivities).

where $\gamma_{S L}, \gamma_{S V}$, and $\gamma_{L V}$ are the interfacial tensions for the solid-liquid, solid-vapor, and liquid-vapor interfaces, respectively.

The result from Shenogina et al. that $G$ could be predicted with only knowledge of the work of adhesion was remarkable in its simplicity. However, this prediction has yet to be rigorously tested with experiments. The experimental work most closely related to these simulations, the study by Ge et al. ${ }^{19}$ considered four interfaces and three were in reasonable agreement with these predictions. ${ }^{20}$ However, those measurements spanned two solids, different chemistry attaching the SAM to the solid, and different alkyl chain lengths. The purpose of the current work is to provide more data but with variation of only the $\omega$-group. In all cases, we have used gold-thiol chemistry so that differences in $G$ between samples could be attributed to the $\omega$-group. The thiol molecules are shown in Table I and are referred to by their $\omega$-group, e.g., $\omega-\mathrm{OH}$. The number of carbon atoms was kept at 11 for four of the five compounds in Table I, but the $\omega$-ester has a much shorter alkyl chain of only three carbon atoms.

Samples, shown schematically in Figure 1, were prepared in the following manner. First, a layer of aluminum was evaporated on a fused silica substrate. The aluminum layer is required for our thermoreflectance measurements of $G$. Next, a layer of gold was evaporated on the aluminum, and the film was immersed overnight in a $1 \mathrm{mM}$ solution of the thiol in ethanol. All thiols were purchased from Sigma-Aldrich. Each sample was rinsed in ethanol followed by water. We found that the quality of the films was improved by immediate immersion of the sample in the ethanol solution after removal from the vacuum chamber used for the aluminum and gold evaporation. Advancing and receding contact angles were measured with a contact angle goniometer (First Ten Ångstroms) at room temperature and are shown in Table I. The data show a range of wettability spanning $25-118^{\circ}$, and the low degree of hysteresis is consistent with homogeneous films.

We use time-domain thermoreflectance ${ }^{3,22}$ (TDTR) to measure the interface thermal conductance, $G$, across the gold-water interfaces. TDTR is a pump-probe optical technique that is an excellent tool for sensitive interface thermal conductance measurements. A Ti:Sapphire femtosecond laser emits pulses with a wavelength of $800 \mathrm{~nm}$ and $\sim 100 \mathrm{fs}$ duration at a repetition rate of $80 \mathrm{MHz}$. The pulses are then split into pump and probe beams that are directed to the surface of the sample, as shown schematically in Fig. 1. The pump beam is modulated at a frequency of $\sim 10 \mathrm{MHz}$, and this beam passes through the transparent fused silica substrate and heats the aluminum surface of the sample. The reflectivity of aluminum ${ }^{23}$ exhibits a relatively large change with respect to temperature near $800 \mathrm{~nm}$, and this thermally induced change in reflectivity is monitored with the timedelayed probe beam. The arrival time of the probe beam at the surface of the sample is adjusted with the use of a mechanical delay stage, and the reflected intensity of the probe

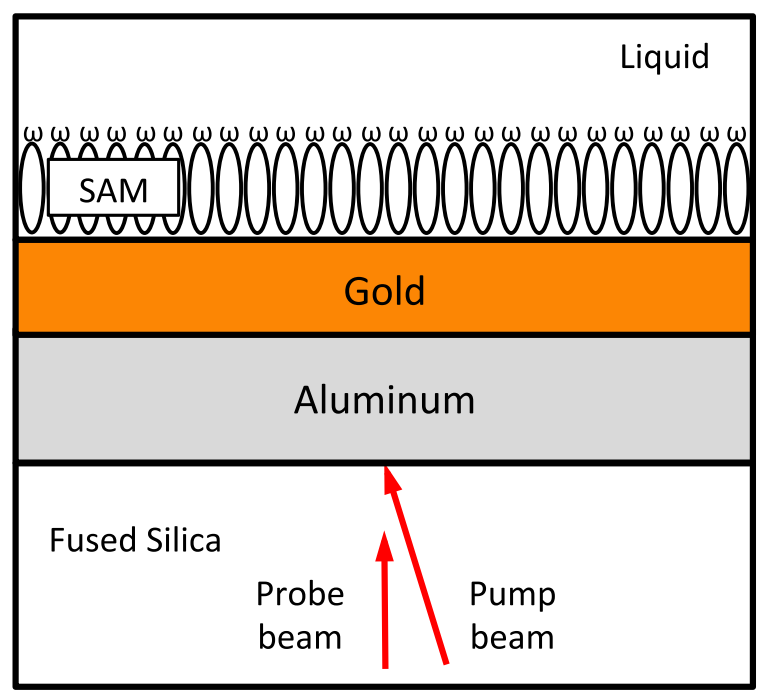

FIG. 1. Schematic diagram of the sample structures. The pump and probe laser beams enter through the transparent fused silica substrate and are reflected from the aluminum film. Aluminum is used as the thermoreflectance layer since it exhibits a relatively large change in reflectivity with temperature at our laser wavelength of $800 \mathrm{~nm}$. Gold is a convenient choice for attaching monolayers since gold-thiol interactions are well understood and allow for the formation of SAMs with a variety of terminal groups (labeled $\omega$ ). 
beam is measured with a photodiode. A lock-in amplifier records the in-phase and out-of-phase voltage produced by the photodiode. Thus, we are able to monitor the thermal response of the surface of the aluminum film for up to $3.5 \mathrm{~ns}$ after the pump beam heats the sample, as shown in Fig. 2 . We use the ratio of the in-phase and out-of-phase voltages, and we compare our experimentally measured ratio with predictions from an analytical thermal model, where $G$ is the only fitting parameter. ${ }^{24}$

The details of the thermal model used to analyze the TDTR data are given by Cahill, ${ }^{24}$ but briefly, the model requires the thickness, heat capacity, and thermal conductivity of each layer. We take the bulk heat capacity for all of the layers in the system. Aluminum and gold film thicknesses are measured with an atomic force microscope after wet chemical etching, while the water and fused silica are treated as infinitely thick. The thermal conductivity of the fused silica substrate is measured separately on a reference sample without a SAM or water, while the thermal conductivity of water is taken from the literature. The thermal conductivities of the gold and aluminum films are determined from electrical conductivity measurements on the films in conjunction with the Wiedemann-Franz law. The interface between the gold and water (including the SAM) is represented in the model as a $1 \mathrm{~nm}$ thick layer, where the ratio of the thermal conductivity to the thickness of the layer gives $G$. This interface conductance is the only fitting parameter in the model, and $G$ is adjusted until the least-squares error between the model and experiment is minimized. Our largest sources of error in extracting $G$ come from uncertainties in the thicknesses and conductivities of the aluminum and gold films. All measurements are repeated on multiple samples prepared separately, and each individual sample is measured at three different locations. Typical variation between measurements on the same sample is less than 5\%, and measurements between samples prepared separately generally exhibit variations of less than $10 \%$.

Our experimentally measured thermal conductance values for the gold-SAM-water samples are shown as a

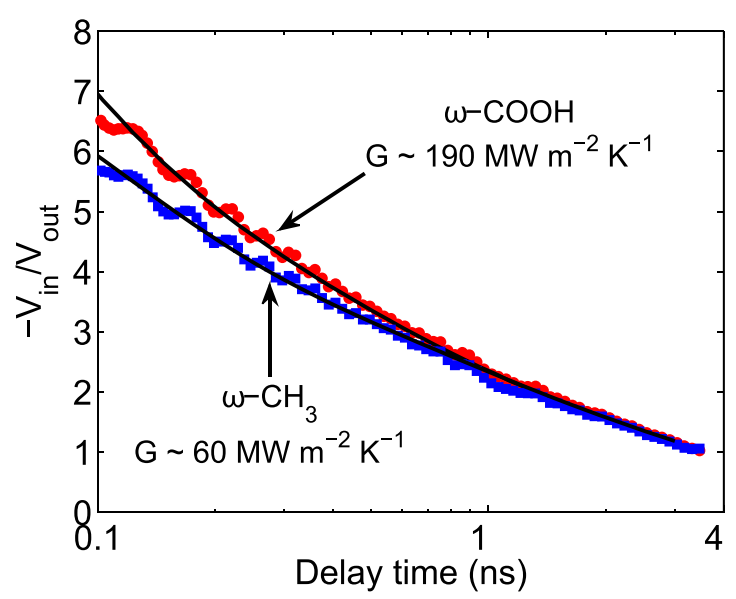

FIG. 2. Comparison of experimental TDTR data with an analytical thermal model. The plot displays the ratio of in-phase to out-of-phase voltage measured by a lock-in amplifier at the photodiode $\left(-V_{\text {in }} / V_{\text {out }}\right)$ as a function of the delay time between the pump and probe beams. The solid circles and squares are experimental data, and the solid lines represent the best fit to our model, where $G$ is the only fitting parameter. The oscillations in the data for $t<500$ ps are due to acoustic echoes in the metal layers. function of the thermodynamic work of adhesion in Figure 3. Clearly, $G$ is a linear function of $W_{\mathrm{SL}}$ for our data, and these measurements are consistent with the predictions by Shenogina et al., ${ }^{20}$ who first stated that $G$ might correlate with the thermodynamic work of adhesion at the interface (Eq. (1)). Our measurements on the hydrophobic SAMs are also consistent with measurements done by Ge et al. ${ }^{19}$ However, we note that we measure a much larger value of $G$ for the same $\omega$-OH SAM than reported by Ge et al. ${ }^{19}$ The origin of this discrepancy is unclear, but our measurement of $G$ for this $\omega$-OH SAM is consistent with our measurement on an $\omega$-COOH SAM with nearly the same contact angle as the $\omega-\mathrm{OH}$.

We caution that the linear relationship between $G$ and $W_{\mathrm{SL}}$ observed here for gold-water interfaces may not be universal for all solid-liquid systems. First, $W_{\mathrm{SL}}$ is a measure of the equilibrium work to separate the solid and liquid from contact to infinity, whereas we expect that transmission of thermally induced vibrations would depend on the stiffness of the interactions between the solid and liquid only near the equilibrium separation distance. Second, this relationship has only been measured for a single planar gold-thiol-alkane- $\omega$ group-water system, and the nature of the interfacial bonding will vary considerably for different solid-liquid systems. We can think of the interface as a set of conductances in series, and perhaps alternate pathways in parallel. We would expect that the observed relationship between $G$ and $W_{\mathrm{SL}}$ would only hold if the connection between the $\omega$-group and water was the limiting conductance. For the particular case studied here, this appears likely. First, alkane thiols with small $\omega$ groups have been previously shown to pack tightly on gold, ${ }^{21}$ thereby excluding solvent from the SAM. Otherwise solvent that penetrates the SAM might provide an alternative conduction pathway. We note that greater $G$ was observed with some of the larger $\omega$-groups, but there is not a uniform

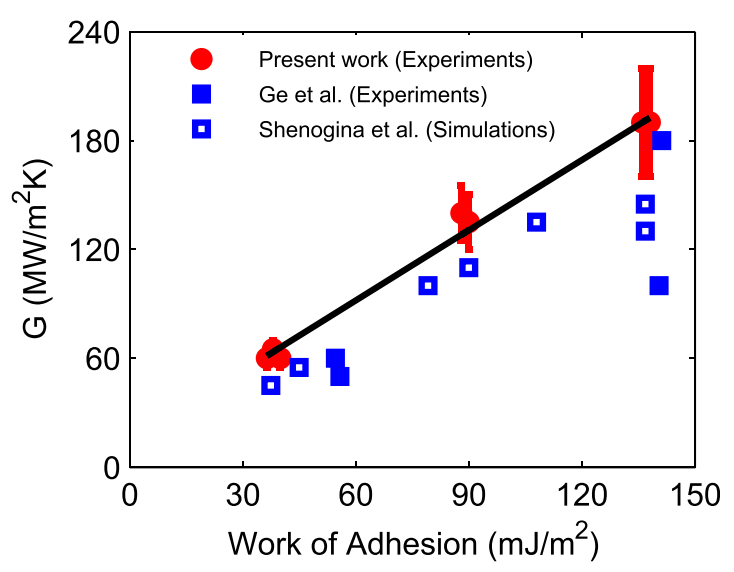

FIG. 3. Measured interface thermal conductance at room temperature as a function of the thermodynamic work of adhesion at the interface, $W_{S L}$. The work of adhesion is calculated from Eq. (1) using the measured value of the surface tension of water $\left(\gamma_{\mathrm{LV}}=72 \mathrm{~mJ} \mathrm{~m}^{-2}\right)$ and the advancing contact angle of water on the SAM as shown in Table I. The solid line is a least squares fit to our data, where $G=1.32 W_{S L}+13\left(\mathrm{R}^{2}=0.987\right)$. The cluster of our data at $W_{S L} \sim 40 \mathrm{~mJ} / \mathrm{m}^{2}$ and $G \sim 60 \mathrm{MW} \mathrm{m} \mathrm{m}^{-2} \mathrm{~K}^{-1}$ represent the three alkanethiols of varying chain length (i.e., three homologues of $n$-undecanethiol with 11,12 , and 18 carbon atoms). The solid square symbols are measurements from Ge et al. (Ref. 19) for various SAMs on Au and Al in water, and the open squares are molecular dynamics simulations from Shenogina et al. (Ref. 20). 
relationship. Second, other parts of the monolayer could provide the limiting conductance. For our SAMs, we did not vary the $\alpha$-group (S-Au bond), so we do not know the importance of this bond, but the relationship between $G$ and the work of adhesion suggests that, in the molecules studied here, the $\alpha$-group is not the limiting thermal resistance. Previous work suggests that conduction through the alkyl chain is ballistic for straight chains and therefore does not limit interface conductance. ${ }^{25-28}$ (We also examine that conclusion below). Finally, water is a peculiar liquid, and it would be wise to examine other substances before making a generalization.

As an alternative to using a thermodynamic parameter $\left(W_{\mathrm{SL}}\right)$ to characterize the range of SAMs used here, we also briefly comment on the bonding between the various $\omega$-groups and the liquid, which is the cause of the variation in $W_{\mathrm{SL}}$. The $\omega-\mathrm{CH}_{3}$ forms only weak van der Waals interactions with the water, whereas the $\omega-\mathrm{OH}$ and $\omega-\mathrm{COOH}$ groups form strong and relatively stiff hydrogen bonds with water. Thus, the high $G$ SAMs have stiff and strong bonds whereas the low $G$ SAM has weak, lower stiffness bonding. The SAMs with moderate values of $G$ have some intermediate ability to bond with water. The $\omega$-pyroll is aromatic, so it can also form quadrupolar bonds with water, and the $\omega$-ester has hydrogen bond acceptor sites that will have some limited access to hydrogen bond to water despite the terminal methyl. A more detailed comparison between bond strength and $G$ could be obtained from MD simulations, such as those performed by Shenogina et al., ${ }^{20}$ where the simulated $G$ could be compared to the input bond forces.

We emphasize that we have observed only a correlation between work of adhesion and $G$; this does not necessarily imply a mechanism because variation in $G$ and in work of adhesion may have the same root cause. We expect that $G$ will depend on an overlap of the density of the thermally stimulated vibrational states of the groups on either side of and at the interface, and measurements of these vibrational states may reveal a more fundamental correlation. It may simply be that the work of adhesion gives some measure of the vibrational states.

Finally, we did test the effect of the alkyl chain length on the interface conductance by measuring three homologues of $n$-undecanethiol with 11,12 , and 18 carbon atoms. The values of $G$ for these three samples fall within a narrow range of $60-65 \mathrm{MW} \mathrm{m} \mathrm{m}^{-2} \mathrm{~K}^{-1}$ with the difference equal to our measurement uncertainty. Therefore, the addition of seven methyl units to the alkane portion of the molecule does not present a thermal resistance that is measureable with our TDTR technique, and possibly the entire alkyl chain does not present a measurable resistance. The contact angles of water on these SAMS were all in the range of $117-119^{\circ}$. Previously, it has been predicted that gauche conformations in an alkane chain should increase the scattering of phonons. ${ }^{26}$ Earlier, infrared spectroscopic measurements ${ }^{29}$ of alkane-thiols with 12-16 carbon atoms showed there is an increasing ratio of gauche:trans conformations with increasing number of methylene units, yet we are not able to resolve any change in $G$ by varying the chain length over the larger range of 11 to 18 carbon atoms. Therefore, our work does not provide support for sufficient gauche scattering of phonons to make this mechanism the limiting conductance.

In summary, the results of interface thermal conductance measurements using time-domain thermoreflectance show that the interface conductance is proportional to the work of adhesion between the solid and liquid for the specific gold-thiol-alkane- $\omega$-group-water system, consistent with ideas presented by Ge et al. ${ }^{19}$ and by Shenogina et al. ${ }^{20}$ We also find that the interface conductance is independent of the length of the alkyl chain in the range of 11-18 carbon units.

This work was supported in part by the Air Force Office of Scientific Research under Grant No. FA9550-10-1-0518. The authors also appreciate support from the Virginia Tech Institute for Critical Technology and Applied Science (ICTAS). We thank Chris Progen and Don Leber for assistance with the SAMs and metal evaporation, respectively.

${ }^{1}$ E. Pop, Nano Res. 3(3), 147 (2010).

${ }^{2}$ W. Kim, R. Wang, and A. Majumdar, Nano Today 2(1), 40 (2007).

${ }^{3}$ D. G. Cahill, W. K. Ford, K. E. Goodson, G. D. Mahan, A. Majumdar, H. J. Maris, R. Merlin, and S. R. Phillpot, J. Appl. Phys. 93(2), 793 (2003).

${ }^{4}$ A. Nitzan, Science 317(5839), 759 (2007).

${ }^{5}$ D. Segal and A. Nitzan, J. Chem. Phys. 117(8), 3915 (2002).

${ }^{6}$ A. K. Roy, B. L. Farmer, V. Varshney, S. Sihn, J. Lee, and S. Ganguli, ACS Appl. Mater. Interfaces 4(2), 545 (2012).

${ }^{7}$ X. Zheng, D. G. Cahill, and J. C. Zhao, Adv. Eng. Mater. 7(7), 622 (2005).

${ }^{8}$ M. G. Kanatzidis, Chem. Mater. 22(3), 648 (2010).

${ }^{9}$ A. J. Minnich, M. S. Dresselhaus, Z. F. Ren, and G. Chen, Energy Environ. Sci. 2(5), 466 (2009).

${ }^{10}$ M. S. Dresselhaus, G. Chen, M. Y. Tang, R. G. Yang, H. Lee, D. Z. Wang, Z. F. Ren, J. P. Fleurial, and P. Gogna, Adv. Mater. 19(8), 1043 (2007).

${ }^{11}$ M. Hu, J. V. Goicochea, B. Michel, and D. Poulikakos, Appl. Phys. Lett. 95(15), 151903 (2009).

${ }^{12}$ C. W. Chang, D. Okawa, A. Majumdar, and A. Zettl, Science 314(5802), $1121(2006)$

${ }^{13}$ G. Huttmann and R. Birngruber, IEEE J. Sel. Top. Quantum Electron. 5(4), 954 (1999).

${ }^{14}$ V. P. Zharov, K. E. Mercer, E. N. Galitovskaya, and M. S. Smeltzer, Biophys. J. 90(2), 619 (2006).

${ }^{15}$ H. Ghasemi and C. A. Ward, J. Phys. Chem. C 115(43), 21311 (2011).

${ }^{16}$ B. Horacek, K. T. Kiger, and J. Kim, Int. J. Heat Mass Transfer 48(8), 1425 (2005).

${ }^{17}$ H. Arnolds, Prog. Surf. Sci. 86(1-2), 1 (2011).

${ }^{18}$ A. Yamakata and M. Osawa, J. Phys. Chem. C 112(30), 11427 (2008).

${ }^{19}$ Z. B. Ge, D. G. Cahill, and P. V. Braun, Phys. Rev. Lett. 96(18), 186101 (2006).

${ }^{20}$ N. Shenogina, R. Godawat, P. Keblinski, and S. Garde, Phys. Rev. Lett. 102(15), 156101 (2009).

${ }^{21}$ C. D. Bain, E. B. Troughton, Y. T. Tao, J. Evall, G. M. Whitesides, and R. G. Nuzzo, J. Am. Chem. Soc. 111(1), 321 (1989).

${ }^{22} \mathrm{~S}$. Huxtable, in Handbook of Instrumentation and Techniques for Semiconductor Nanostructure Characterization, edited by R. Haight, F. M. Ross, and J. B. Hannon (World Scientific Publishing Co., Hackensack, NJ, 2012), Vol. 2, p. 587.

${ }^{23}$ Y. Wang, J. Park, Y. K. Koh, and D. G. Cahill, J. Appl. Phys. 108, 043507 (2010).

${ }^{24}$ D. G. Cahill, Rev. Sci. Instrum. 75(12), 5119 (2004).

${ }^{25}$ J. C. Duda, C. B. Saltonstall, P. M. Norris, and P. E. Hopkins, J. Chem. Phys. 134(9), 094704 (2011).

${ }^{26}$ K. Sasikumar and P. Keblinski, J. Appl. Phys. 109(11), 114307 (2011).

${ }^{27}$ R. Y. Wang, R. A. Segalman, and A. Majumdar, Appl. Phys. Lett. 89(17), 173113 (2006).

${ }^{28}$ Z. H. Wang, J. A. Carter, A. Lagutchev, Y. K. Koh, N. H. Seong, D. G. Cahill, and D. D. Dlott, Science 317(5839), 787 (2007).

${ }^{29}$ L. Bertilsson and B. Liedberg, Langmuir 9(1), 141 (1993). 